\title{
The Italian Society for Rheumatology clinical practice guidelines for the diagnosis and management of fibromyalgia Best practices based on current scientific evidence
}

\author{
${ }^{*}$ A. Ariani ${ }^{1,2}$, L. Bazzichi ${ }^{3}$, P. Sarzi Puttini ${ }^{4}$, F. Salaffi ${ }^{5}$, M. Manara ${ }^{1,6}$, I. Prevete ${ }^{1,7}$, \\ A. Bortoluzzi ${ }^{1,8}$, G. Carrara ${ }^{1}$, C.A. Sciré1,8, *N. Ughi ${ }^{1,9},{ }^{\star}$ S. Parisi ${ }^{1,10}$ \\ ${ }^{*} A A, S P$ and $N U$ contributed equally to the paper \\ ${ }^{1}$ Epidemiology Unit, Italian Society for Rheumatology, Milan, Italy; '2Dipartimento di Medicina, Unità di \\ Medicina Interna e Reumatologia, Azienda Ospedaliero-Universitaria di Parma, Italy; ${ }^{3}$ Unità di Reumatologia, \\ Azienda Ospedaliero Universitaria Pisana, Pisa, Italy; ${ }^{~}$ Unità di Reumatologia, ASST Fatebenefratelli-Sacco, \\ Università di Milano, Italy; ${ }^{5}$ Clinica Reumatologica, Ospedale 'Carlo Urbani', Università Politecnica delle \\ Marche, Jesi (AN), Italy; ${ }^{6}$ Reumatologia Clinica, Centro Specialistico Ortopedico-Traumatologico Gaetano Pini \\ CTO, ASST Gaetano Pini, Milano, Italy; ' Unità di Reumatologia, Azienda Ospedaliera San Camillo-Forlanini, \\ Roma, Italy; ${ }^{\circledR}$ Dipartimento di Scienze Mediche, Sezione di Reumatologia, Università di Ferrara, Azienda \\ Ospedaliero-Universitaria Sant'Anna, Cona (FE), Italy; ' Division of Rheumatology, ASST Grande \\ Ospedale Metropolitano Niguarda, Milano, Italy; ${ }^{10}$ Unità di Reumatologia, Azienda Ospedaliera Città \\ della Salute e della Scienza di Torino, Italy
}

\section{SUMMARY}

Fibromyalgia or fibromyalgia syndrome (FMS) is defined as a central sensitization syndrome characterized by the dysfunction of neurocircuits detecting, transmitting and processing nociceptive stimuli; the prevalent manifestation is musculoskeletal pain. In addition to pain, there are multiple accompanying symptoms, in common with other algo-dysfunctional syndromes, which are reflected in a broad spectrum of somatic, neurocognitive and neuro-vegetative manifestations. An evidence-based approach is essential in FMS management, in order to improve patient health and to reduce its social burden. Since in the last ten years new international guidelines for clinical practice (Clinical Practice Guidelines or CPGs) concerning FMS diagnosis and pharmacological/ non-pharmacological management have been published, the Italian Society of Rheumatology (SIR) has decided to adapt them to the Italian national setting.

The framework of the Guidelines International Network Adaptation Working Group was adopted to identify, appraise (AGREE II), synthesize, and customize the most recent CPGs on FMS to the needs of the Italian healthcare context. A working group of rheumatologists from SIR epidemiology unit and FMS experts identified relevant clinical questions to guide the systematic review of the literature. The target audience of these CPGs included physicians and healthcare professionals who manage FMS. The adapted recommendations were finally assessed by an external multidisciplinary panel.

From the systematic search in databases (Pubmed/Medline, Embase) and grey literature, 6 CPGs were selected and appraised by two independent raters. The combination of the scientific evidence underlying the original CPGs with expert opinion lead to the development of 17 recommendations. The quality of evidence for each recommendation was reported and their potential impact on clinical practice was assessed.

These SIR recommendations are expected to be a valuable aid in the diagnosis and treatment of FMS, as they will contribute to disseminate the best practice on the basis of the current scientific evidence.

Key words: Clinical Practice Guideline, recommendations, fibromyalgia, diagnosis; treatment.

Reumatismo, 2021; 73 (2): 89-105

\section{INTRODUCTION}

ibromyalgia or fibromyalgia syndrome (FMS) is defined as a central sensitization syndrome characterized by the dysfunc- tion of neurocircuits detecting, transmitting and processing nociceptive stimuli; the prevalent manifestation is musculoskeletal pain (1). In addition to pain, there may be multiple accompanying symptoms in com-
Corresponding author: Alarico Ariani Department of Medicine, Internal Medicine and Rheumatology Unit, Azienda Ospedaliero Universitaria di Parma Via Gramsci, 14 - 43100 Parma (PR) Italy E-mail: aariani@ao.pr.it 
mon with other algo-dysfunctional syndromes, such as fatigue, sleep disturbances, cognitive disturbances (e.g. attention or memory deficits), mental problems (e.g. anxiety, depression) and, in general, a broad spectrum of somatic, neurocognitive and neuro-vegetative symptoms. The prevalence is between $2 \%$ and $8 \%$, while the incidence is between 7 and 11 cases/year per 1000 people (1-3). The epidemiological estimates vary according to the criteria used for FMS diagnosis. For example, the prevalence of FMS in the general population was $1.7 \%$ following the criteria of the 1990 American College of Rheumatology (ACR), but raised up to $5.4 \%$ when the modified version of the 2010 ACR criteria was considered (4). FMS is more frequent in women than men and it can occur at any age.

Although the etiology is not yet fully understood and the pathophysiological framework not clearly delineated (2), some pathogenetic hypotheses concerning the responsible for the centralization of pain mechanism have been proposed. An alteration of central nervous system (CNS) pain control mechanisms seems to be involved in both hyperalgesia and other symptoms (memory disturbances, fatigue and depression). In a proportion of FMS patients there is a reduction of pain modulation ability through the descending pathways: in particular serotonergic-noradrenergic activity seems to be compromised. Therapeutic benefits provided by serotonin and norepinephrine reuptake inhibitors (SNRIs) support this hypothesis. Moreover, FMS patients have a reduced binding capacity of opioid receptors from different brain regions with a potentially relevant pain modulating role (5). Glial cells participate in pain modulation, especially neuropathic pain. Their activation, through pro-inflammatory cytokines (TNF, IL-6, IL-8) and opioids, is not mediated by the respective receptors, but by the toll-like receptor 4 (TLR-4), recently identified as a competing element to chronic pain (6, $7)$. Another hypothesis is the increase of glutamate levels in the cerebrospinal fluid of FMS patients, while gamma aminobutyric acid (GABA) levels are reduced (8,
9). The role of the anterior limb cortex on pain modulation is not yet fully clarified. It is supposed that inhibitory neurotransmitters (i.e. GABA and opioids) release reduce neuronal excitability of the ventromedial rostral marrow with a downward pain modulation (10). In FMS, cerebral perfusion tomoscintigraphy (SPECT) and functional magnetic resonance imaging (fMRI) showed perfusion anomalies and high activity in the somatosensory cortex, while the activity was reduced in the frontal cortex, in the cingulum, in the temporal and cerebellar cortex (11-13). Finally, the phenomenon of the temporal summation of pain (or 'windup'), supported by the repetitive stimulation of the nociceptive fibers, may contribute to FMS pathogenesis (14, $15)$. The above-mentioned hypotheses suggest that a predominant role can be played both by an alteration of the CNS pain pathways and by small peripheral fibers inflammation (16).

The heterogeneous set of pathogenetic hypotheses prompted the experts to identify FMS diagnostic criteria based not only on characteristic symptoms, but also on the exclusion of other pathologies (2, 17, 18). Similarly, the management and the treatment are the main topic of several studies whose results have allowed the development of clinical practice guidelines (CPGs). The most recent CPGs have been developed by scientific societies with reference to different healthcare systems. So, it is not certain that they are easily implementable in the Italian context.

In light of the absence of Italian recommendations on FMS so far, new CPGs were developed on behalf of the Italian Society of Rheumatology (SIR) for the Italian healthcare setting.

\section{Objective}

These guidelines aim to provide evidencebased recommendations adapted to the national context on the diagnosis and the treatment of patients with FMS in Italy.

\section{Target patient population}

Adult patients (age $\geq 18$ years) with suspected or defined diagnosis of FMS formu- 
lated by a rheumatologist or other specialist or primary care physician.

\section{Target users}

The recommendations are addressed to physicians (rheumatologists, physiatrists and general practitioners) and health professionals who are involved in the management of FMS in primary care or in hospital, community and academic practice settings, patients, policy makers and those responsible for commissioning care for patients with FMS in the Italian National Health Service (NHS).

\section{What is covered}

These recommendations cover the different stages of FMS patient management, including diagnosis, pharmacological and non-pharmacological treatment and disease monitoring over time.

\section{Funding}

These recommendations matched no specific funding from any bodies in the public, commercial sectors or non-profit organization. Non-economic support, such as meeting spaces and secretarial services, was provided by SIR.

\section{MATERIALS AND METHODS}

\section{Approach to guideline development}

The development, updating and application of high-quality recommendations and guidelines in clinical practice require a consistent use of time and resources, as well as significant investments to promote their diffusion. In order to limit the de novo development of local guidelines, to improve efficiency and to promote the adoption of updated guidelines, a systematic approach based on the framework of the Guidelines International Network Adaptation Working Group (http://www.gin. net) and the ADAPTE collaboration (19, 20) was adopted to identify, appraise, synthesize, and customize the existing international guidelines to the needs of the Italian healthcare context.

\section{Assembly of the Working Group}

On behalf of SIR, a working group consisting of 7 rheumatologists (AA, SP, NU,
CAS, IP, AB, MM) and a biostatistician (GC) from the SIR epidemiology research unit were responsible of CPG methodology and development. Three rheumatologists with experience in FMS (LB, FS, PSP) were involved in each phase of the guideline development by attending a working group at the $55^{\text {th }}$ SIR National Meeting, contributing to e-mail discussions and participating to a web-meeting.

\section{Stakeholder involvement}

The draft of these recommendations was revised and rated by an external multidisciplinary commission with 12 members including 7 rheumatologists, an anesthesiologist, a physiatrist, a physiotherapist, a professional nurse and a representative of the patients' organization at the invitation of the SIR. These recommendations were developed without any input from, or cooperation with any pharmaceutical company.

\section{Defining the scope}

The working group defined the objective of the guidelines and developed by consensus a list of key clinical questions about FMS management to be addressed. Seventeen clinical questions defined (Table I) guided the systematic literature search.

\section{Inclusion and exclusion criteria}

Consensus statements and CPGs with recommendations for FMS endorsed by international and national scientific societies, published in English or in Italian between $1^{\text {st }}$ January 2009 and $31^{\text {st }}$ December 2018, were included. Published studies which did not provided guidelines or consensus statements including randomized controlled trials (RCT) and uncontrolled trials, observational studies, editorials, commentaries, conference abstracts and narrative/ systematic reviews were excluded. CPGs and consensus statements in languages other than English and Italian, which were non-original (i.e. duplicated, adapted or updated previous recommendations) and/ or reported with poor methodology, and did not answer to the key health questions were excluded as well. 
Table I - Key questions regarding the overall management of patients with fibromyalgia syndrome (FMS). Seventeen health questions guided the systematic review and the adapted recommendations development.

\begin{tabular}{|c|c|c|}
\hline N. & Text of the health question & N. recommendation \\
\hline \multicolumn{3}{|c|}{ GENERAL QUESTIONS } \\
\hline 1 & What kind of approach is needed to implement? & 1 \\
\hline 2 & What is the target of the therapy? & ॥ \\
\hline \multicolumn{3}{|c|}{ DIAGNOSIS } \\
\hline 3 & What classification criteria to use? & III \\
\hline 4 & What clinical symptoms and signs are related to FMS? & IV \\
\hline 5 & What laboratory and instrumental tests to perform? & V \\
\hline 6 & $\begin{array}{l}\text { What information and/or support should be provided to the patient } \\
\text { at the time of diagnosis? }\end{array}$ & $\mathrm{VI}$ \\
\hline \multicolumn{3}{|c|}{ PHARMACOLOGIC TREATMENT } \\
\hline 7 & What should the pharmacological strategy be, in general? & VII \\
\hline 8 & Which analgesic/opioid drugs are useful in the treatment of FMS? & VIIII \\
\hline 9 & What anticonvulsant drugs are useful in the treatment of FMS? & $\mathrm{IX}$ \\
\hline 10 & What antidepressant drugs are useful in the treatment of FMS? & $\mathrm{X}$ \\
\hline 11 & What drugs are useful in FMS insomnia? & $\mathrm{XI}$ \\
\hline \multicolumn{3}{|c|}{ NON PHARMACOLOGIC TREATMENT } \\
\hline 12 & What should the non-drug strategy be, in general? & XII \\
\hline 13 & What physical therapies can benefit the patient with FMS? & XIII \\
\hline 14 & What psychological therapies can benefit the patient with FMS? & XIV \\
\hline 15 & What alternative therapies can benefit the patient with FMS? & $\mathrm{XV}$ \\
\hline \multicolumn{3}{|c|}{ FOLLOW-UP } \\
\hline 16 & What clinical or clinimetric elements should be considered during monitoring? & $\mathrm{XVI}$ \\
\hline 17 & How long should drug and non-drug therapy be continued? & $\mathrm{XVII}$ \\
\hline
\end{tabular}

Results Medline, Embase

1 January 2009 - 31 December 2018 $(n=135)$

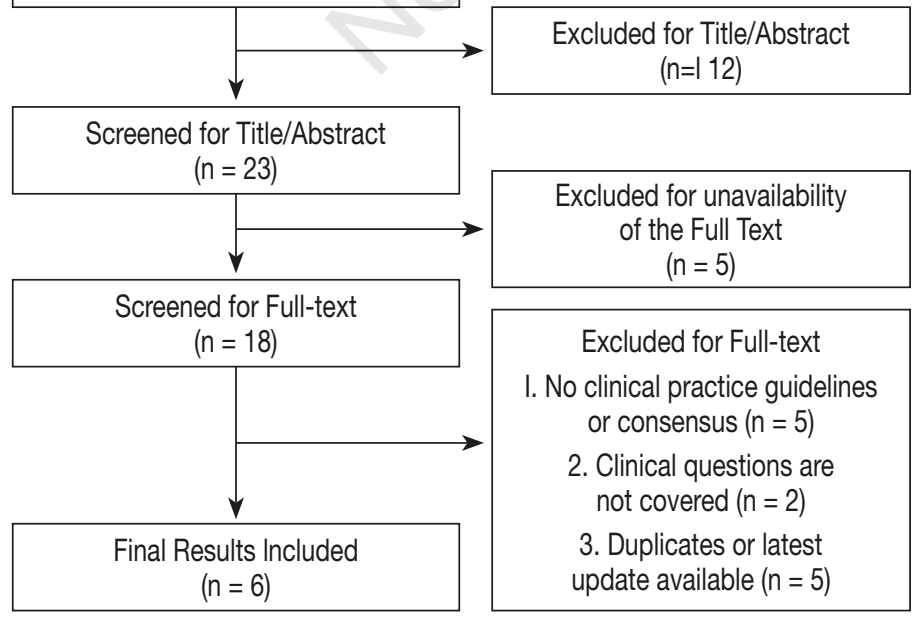

Figure 1 - Steps in the systematic review of guidelines on diagnosis and treatment of FMS.

\section{Search strategy}

The research was performed on Medline via PubMed and Embase. The results were assessed and selected by two independent reviewers (A.A., S.P.) and the disagreements were solved by consensus. Figure 1 shows the flow of research results.

\section{Appraisal of guideline quality}

Guideline quality was assessed by two raters (A.A., M.M.) using the on-line Appraisal of Guidelines Research and Evaluation (AGREE) II instrument (21). The final AGREE score was not considered as criteria for exclusion.

\section{Level of evidence and strength of recommendation}

The included CPGs adopted different systems of classification of evidence. In order to reconcile these differences, the classification system of each CPG was trans- 
Table II - Guidance to categories of evidence and strength of recommendations based on the Oxford Levels of Evidence.

\begin{tabular}{|c|l|}
\hline Category & Evidence \\
\hline 1 & $\begin{array}{l}\text { From meta-analysis of randomised controlled trials or from at least one randomised } \\
\text { controlled trial }\end{array}$ \\
\hline 2 & From at least one controlled study without randomisation or from at least one cohort study \\
\hline 3 & From at least one case-control study \\
\hline 4 & From case-series or poor-quality cohort and case-control studies \\
\hline 5 & From expert committee reports or opinions and/or clinical experience of respected authorities \\
\hline
\end{tabular}

lated reporting the level of evidence and strength of the corresponding recommendation according to the Oxford Levels of Evidence (www.cebm.net/oxford-centreevidence-based-medicine-levels-evidencemarch-2009/) (Table II) (22). Where a discrepancy between classifications was found, the level of the recommendation based on the most recent scientific evidence was taken into account.

\section{Evidence framework and development of recommendations}

The descriptive characteristics of included guidelines (guideline developer, topic, country, language, publication year, end-of-search date, grading systems) and AGREE scores were summarized in tables (not shown). For each clinical question, tables with guideline characteristics, recommendations, AGREE summary scores, and level of evidence and strength of recommendation according to the original grading system were prepared. Each final recommendation was developed by endorsement or adaptation and rewording of the source recommendations. The final CPG were reported in accordance with the AGREE checklist (21). Further details on the methodology were previously described (23).

\section{External review}

The draft of the recommendations was sent to stakeholders $(n=18)$. An online survey was conducted via Google Forms between $30^{\text {th }}$ July 2019 and $30^{\text {th }}$ August 2019 and the responses $(n=12,68 \%$ response rate) were considered for the final statements of the recommendations.

\section{RESULTS}

\section{Key to understanding this guidance}

Each recommendation is presented with a level of evidence and strength of recommendation and is accompanied by a supporting text that is structured as follows: Summary of guidelines: a synthesis of recommendations included in the original guidelines on FMS identified from the systematic review.

Table III - The final set of 17 recommendations for the management of FMS with respective level of evidence.

\begin{tabular}{|c|l|c|}
\hline $\mathbf{N}$ & Recommendation & $\begin{array}{c}\text { Level of } \\
\text { Evidence }\end{array}$ \\
\hline 1 & $\begin{array}{l}\text { FMS requires an overall assessment of pain, function, comorbidities and the psychosocial context. In general, FMS } \\
\text { management should be based on a gradual approach (level 4, grade D). Diagnosis, assessment of severity and } \\
\text { coordination of treatment should be performed by a rheumatologist with knowledge and experience in FMS treatment. } \\
\begin{array}{l}\text { Patients with mild FMS can be managed in a primary care setting with an experienced healthcare professional and } \\
\text { patient support groups (level 3, grade C). A specialist continuous management should be reserved for patients who } \\
\text { have failed the early stages of treatment or who have complex comorbidities (level 5, grade D). For selected subjects, a } \\
\text { multidisciplinary team that includes sleep specialists, nutritionists or psychologists (level 1, grade A) may be necessary. } \\
\text { Since the treatment strategy encompasses the principles of self-management and it uses a multi-modal method (level 1, } \\
\text { grade A), a patient tailored approach is recommended, with careful and regular monitoring, especially in the early stages } \\
\text { (level 5, grade D) }\end{array}\end{array}$ \\
\hline
\end{tabular}




\begin{tabular}{|c|c|c|}
\hline $\mathbf{N}$ & Recommendation & $\begin{array}{l}\text { Level of } \\
\text { Evidence }\end{array}$ \\
\hline 2 & $\begin{array}{l}\text { FMS management should aim to improve health-related quality of life by balancing the benefits and risks of treatment } \\
\text { (level } 4 \text {, grade D). } \\
\text { At the beginning of treatment, patients must be encouraged to identify specific objectives regarding the state of health } \\
\text { and quality of life, and to evaluate their achievement during the follow-up (level } 5 \text {, grade D). }\end{array}$ & $4-5$ \\
\hline 3 & $\begin{array}{l}\text { The clinical diagnosis is based on the presence of the peculiar symptoms, lasting for at least three months, excluding } \\
\text { those ones that related to other diseases (level 5, grade D). The } 2016 \text { review of the American College of Rheumatology } \\
\text { (ACR) FMS diagnostic criteria (2011/2010) is useful in the initial assessment in order to support a clinical diagnosis; } \\
\text { however, it should be taken into account that symptoms vary over time (level 3, grade B). } \\
\text { Physical examination should be in the normal range with the exception of hypersensitivity to soft tissue pressure (level } 5 \text {, } \\
\text { grade D); nevertheless, the examination of 'tender points' according to the } 1990 \text { ACR diagnostic criteria has little clinical } \\
\text { relevance and does not confirm a diagnosis of FMS (level 5, grade D). }\end{array}$ & $3-5$ \\
\hline 4 & $\begin{array}{l}\text { The pivotal symptoms are: } \\
\text { a) chronic and widespread musculoskeletal pain; } \\
\text { b) fatigue and asthenia; } \\
\text { c) sleep disorders; } \\
\text { d) neurocognitive disorders. } \\
\text { Psycho-affective alterations (anxiety, depression, etc.) may be related with a wide range of somatic and neurovegetative } \\
\text { symptoms with different variability (level 5, grade D). Healthcare professionals should be aware that some medical or } \\
\text { psychological conditions may arise with pain and that patients with other medical conditions may have an associated } \\
\text { FMS (level 5, grade D). }\end{array}$ & 5 \\
\hline 5 & $\begin{array}{l}\text { FMS should be diagnosed as a clinical construct without any confirmatory laboratory tests (level } 5 \text {, grade D). Repeated } \\
\text { examinations after diagnosis should be avoided, unless guided by the onset of new symptoms or semeiotic findings (level } \\
5 \text {, grade D). Any further laboratory or radiographic analysis should depend on the individual patient's clinical evaluation, } \\
\text { which may suggest some other medical condition (level } 5 \text {, grade D). }\end{array}$ & 5 \\
\hline 6 & $\begin{array}{l}\text { After FMS diagnosis, the patient should be informed about the recommended and non-recommended treatment } \\
\text { measures (level } 4 \text {, grade A). Healthcare professionals should be educated about the pathogenesis, empathetic, open } \\
\text { and honest, without negative attitudes and pursuing the realization of a shared decision-making process (level } 3 \text {, grade } \\
\text { D). The patient should be informed that FMS is a disease characterized by alterations of the functional sphere and there } \\
\text { is no clear evidence of organic damage. Healthcare professionals should confirm the legitimacy of the disorder, provide } \\
\text { information on the long-term prognosis (i.e. quoad-vitam assessment) and clearly explain the symptoms to the patient } \\
\text { (level 3, grade D). } \\
\text { The patient's ability to alleviate these symptoms through coping activities and strategies should be emphasized, along } \\
\text { with the fact that the outcome is often favorable, even if the symptoms may vary over time (level 3, grade B). Patient } \\
\text { associations may play an active role in promoting educational and support activities (e.g. self-help groups, information } \\
\text { material) (level 5, grade D). }\end{array}$ & $3-5$ \\
\hline 7 & $\begin{array}{l}\text { Physicians should choice the pharmacological strategy (including drugs' combination) according to symptoms and paying } \\
\text { particular attention to drug interactions (level } 5 \text {, grade D). Pharmacological treatments should be started at low doses with } \\
\text { a progressive and prudent increase in the dosage, in order to reduce the poor tolerance and the appearance of possible } \\
\text { side effects (level 5, grade D). } \\
\text { In more complex cases, poorly responsive to standard therapies, it is advisable to adopt a 'multi-modal' approach } \\
\text { (physical activity with at least psychotherapeutic support), to be shared with the patient (level 1, grade A). }\end{array}$ & $1-5$ \\
\hline 8 & $\begin{array}{l}\text { According to WHO step-up analgesic scale, paracetamol is useful in some patients; clinicians should be aware of the } \\
\text { dose necessary to the pharmacological effect and the dose causing a toxic effect (level 5, grade D). } \\
\text { A therapeutic attempt with weak opioids, especially tramadol (level 1, grade A), should be reserved for patients with } \\
\text { moderate to severe pain who do not respond to other treatment modalities (level } 2 \text {, grade D). } \\
\text { Doctors should closely monitor pharmacological action, with particular reference to drug-dependent side effects or } \\
\text { behavioral disorders (level 5, grade D). } \\
\text { Opioid use should be discouraged in the absence of symptoms improvement (level 5, Grade D). }\end{array}$ & $1-5$ \\
\hline 9 & $\begin{array}{l}\text { Anticonvulsant drugs, in particular pregabalin, are useful for their pain modulating properties; treatment should } \\
\text { start with the lowest possible dose, followed by a progressive increase in dosage, with attention to adverse events } \\
\text { (level } 1 \text {, grade A). }\end{array}$ & 1 \\
\hline 10 & $\begin{array}{l}\text { In FMS treatment, serotonin reuptake inhibitors (fluoxetine and paroxetine) and norepinephrine (duloxetine) or tricyclic } \\
\text { antidepressant drugs (amitriptyline) (level 1, grade A) may be used. }\end{array}$ & 1 \\
\hline 11 & $\begin{array}{l}\text { Treatment with cyclobenzaprine (level 1, grade A) and cannabinoids (level 3, grade C) may be considered in FMS patient, } \\
\text { especially in the context of significant sleep disturbances. }\end{array}$ & $1-3$ \\
\hline
\end{tabular}




\begin{tabular}{|c|c|c|}
\hline $\mathbf{N}$ & Recommendation & $\begin{array}{l}\text { Level of } \\
\text { Evidence }\end{array}$ \\
\hline 12 & $\begin{array}{l}\text { Non-pharmacological strategies providing for the patients' active participation should be an integral part of FMS } \\
\text { therapeutic approach (level 1, grade A). Physicians should encourage patients to pursue a regular lifestyle, gradually } \\
\text { increasing physical activity through stimuli and/or to maintain or improve function and promote self-management by } \\
\text { multi-modal therapy (level 4, grade D). Physicians should inform patients of the negative impact that the psychological } \\
\text { distress associated with FMS can generate (level 3, grade D) and therefore promote psychological assessment or } \\
\text { counseling (level 5, grade C). }\end{array}$ & $1-5$ \\
\hline 13 & $\begin{array}{l}\text { Physical therapies (adapted to patient individual performance level) should be considered in FMS, such as aerobic } \\
\text { resistance training; strengthening exercise; water activity/water jogging; thermal therapy (bath in thermal springs) (level 1, } \\
\text { grade A). }\end{array}$ & 1 \\
\hline 14 & $\begin{array}{l}\text { Psychological therapies to be considered may include: } \\
\text { a) behavioral-cognitive therapy and occupational therapy, including patient education, even for a short period (level } 1 \text {, } \\
\text { grade A); } \\
\text { b) hypnosis, guided imagination or therapeutic writing (level 3, grade C). }\end{array}$ & $1-3$ \\
\hline 15 & $\begin{array}{l}\text { Non-conventional therapies to be considered may be: } \\
\text { - meditative movement therapies (qigong, yoga, tai chi), mindfulness-based stress reduction program and relaxation } \\
\text { training (combined with exercise) (level 1, grade A). } \\
\text { - acupuncture (level 1, grade A). } \\
\text { - hydrotherapy (level 1, grade C). }\end{array}$ & 1 \\
\hline 16 & $\begin{array}{l}\text { Clinical follow-up should rely on the judgment of the rheumatologist, with more frequent visits during the initial phase or } \\
\text { until the symptoms stabilization (level } 5 \text {, grade E). The development of a new symptom may require a clinical evaluation } \\
\text { in order to exclude another disease (level 5, grade E). Patient objectives and achievement levels should be recorded as } \\
\text { a useful strategy for outcome (level 5, grade E). Physicians should take into consideration that factors such as passivity, } \\
\text { lack of self-control and mood disturbance may negatively influence the outcomes (level } 5 \text {, grade E). Tender points } \\
\text { examination should not be an outcome measure (level 3, grade C). }\end{array}$ & $3-5$ \\
\hline 17 & $\begin{array}{l}\text { Treatment benefits should be regularly evaluated by patients and physicians as therapy should be continued only if } \\
\text { positive effects are experienced. In the case of response to drug therapy, after an appropriate duration of treatment, a } \\
\text { gradual withdrawal (level 2, grade A) should be considered. } \\
\text { Patients who experience improvement with aerobic endurance training should constantly implement it (level 1, grade A). } \\
\text { Physicians should encourage patients to work providing, if necessary, recommendations about maintaining the optimal } \\
\text { productivity as the outcome is generally more favorable for those who are employed (level } 3 \text {, grade D). FMS patients on } \\
\text { long-term sick leave should join appropriate rehabilitation program aimed at improving function, including returning to } \\
\text { work if possible (level 5, grade D). } \\
\text { For long-term therapy, procedures that can be carried out independently (e.g., individual adaptation to resistance and/or } \\
\text { strength training, stretching or heat therapy) should be self-managed by patients (level } 2 \text { grade A). }\end{array}$ & $1-5$ \\
\hline
\end{tabular}

Recommendation/supporting evidence: specific source guidelines that were used for adaptation (from the most recent).

Evidence to recommendation: results of the panel's discussion of the adapted recommendation with regards to further specifications and comments on the sources used to develop the recommendation.

\section{Recommendations}

Six original CPGs (24-29) were selected and used to accomplish the final set of 17 recommendations (Table III).

\section{RECOMMENDATION 1}

FMS requires an overall assessment of pain, function, comorbidities and the psy- chosocial context. In general, FMS management should be based on a gradual approach (level 4, grade D). Diagnosis, assessment of severity and coordination of treatment should be performed by a rheumatologist with knowledge and experience in FMS treatment. Patients with mild FMS can be managed in a primary care setting with an experienced healthcare professional and patient support groups (level 3, grade C).

A specialist continuous management should be reserved for patients who have failed the early stages of treatment or who have complex comorbidities (level 5 , grade D). 
For selected subjects, a multidisciplinary team that includes sleep specialists, nutritionists or psychologists (level 1, grade A) may be necessary. Since the treatment strategy encompasses the principles of self-management and it uses a multi-modal method (level 1, grade A), a patient tailored approach is recommended, with careful and regular monitoring, especially in the early stages (level 5, grade D).

Summary of guidelines. All recommendations agree that FMS patients should be taken over by a multidisciplinary team. The rheumatologist, indispensable for differential diagnosis, identifies, on the basis of FMS severity and therapeutic path to which the patient is candidate, which professional figures to involve. Reliance on non-rheumatological specialists may be considered for less complicated management cases.

Recommendation/supporting evidence. Emilia-Romagna region (Italy) 2017, EULAR 2016, Canadian 2013, German 2012.

Evidence to recommendation. The Fibromyalgia Working Group set up by the Italian Ministry of Health identifies the rheumatologist as the central specialist figure for the diagnosis of FMS (30). The role of the rheumatologist is also to define FMS severity, supervise pharmacological management, coordinate any specialist consultations (e.g. dietician, psychiatrist or pain therapist) and establish followup procedures. Taking into account the recommendations regarding non-pharmacological therapies, it is advisable to involve physiatrists, psychologists and physiotherapists in the management of patients with FMS. For the complexity of the pathology and the therapeutic strategies, it is suggested that the management by the general practitioner in cooperation with the patients' associations, should only be reserved for patients with mild disease or not complicated by significant comorbidities.

External reviewers median score $8 / 10$ ( $75 \%$ of scores $\geq 7$ ).

\section{RECOMMENDATION 2}

FMS management should aim to improve health-related quality of life by balancing the benefits and risks of treatment (level 4 , grade D).

At the beginning of treatment, patients must be encouraged to identify specific objectives regarding the state of health and quality of life, and to evaluate their achievement during the follow-up (level 5 , grade D).

Summary of guidelines. The FMS treatment aims are listed only in the most recent selected CPGs. Quality of life can be compromised not only by FMS, but also by the side effects of improper drug treatment. For this reason, the objectives include a tailored therapy starting from non-pharmacological strategies.

Recommendation/supporting evidence. EULAR 2016, Canadian 2013.

Evidence to recommendation. The importance of improving the quality of life, working skills and productivity is confirmed by the fibromyalgia working group set up at the Italian Ministry of Health (30). It is up to the rheumatologist, guided by the patient's symptoms, to decide how often to evaluate the achievement and maintenance of the objectives.

External reviewers median score 9/10 (91\% of scores $\geq 7$ ).

\section{RECOMMENDATION 3}

The clinical diagnosis is based on the presence of the peculiar symptoms, lasting for at least three months, excluding those related to other diseases (level 5, grade D).

The 2016 review of the American College of Rheumatology (ACR) FMS diagnostic criteria $(2011 / 2010)$ is useful in the initial assessment in order to support a clinical diagnosis; however, it should be taken into account that symptoms vary over time (level 3, grade B).

Physical examination should be in the normal range with the exception of hypersensitivity to soft tissue pressure (level 5 , grade D); nevertheless, the examina- 
tion of 'tender points' according to the 1990 ACR diagnostic criteria has little clinical relevance and does not confirm a diagnosis of FMS (level 5, grade D).

Summary of guidelines. All the selected guidelines agree that FMS diagnosis should be based on well-defined clinical criteria. The 2010 ACR diagnostic criteria, modified in 2011, are considered the most suitable in confirming clinical diagnosis. The guidelines agree in not assigning any diagnostic role to the presence of 'tender points'.

Recommendation/supporting evidence. Emilia-Romagna 2017, Canadian 2013, German 2012.

Evidence to recommendation. Experts consider the diagnostic criteria revised in 2016 by Wolfe et al. (31) better than those proposed by the ACR in 2011 and endorsed by all the selected CPGs (32). Note that the diagnosis of FMS is independent of the presence of any comorbidity.

External reviewers median score $8 / 10$ ( $82 \%$ of scores $\geq 7$ ).

\section{RECOMMENDATION 4}

The pivotal symptoms are:

a) chronic and widespread musculoskeletal pain;

b) fatigue and asthenia;

c) sleep disorders;

d) neurocognitive disorders.

Psycho-affective alterations (anxiety, depression, etc.) may be related with a wide range of somatic and neurovegetative symptoms with different variability (level 5, grade D).

Healthcare professionals should be aware that some medical or psychological conditions may arise with pain and that patients with other medical conditions may have an associated FMS (level 5, grade D).

Summary of guidelines. In the selected guidelines there is a perfect agreement in listing the musculoskeletal and neurovegetative symptoms related to FMS. However, the clinicians should always consider the possibility of association with other pathological conditions (e.g vitamin D deficiency).
Recommendation/supporting evidence. Emilia-Romagna 2017, Canadian 2013, German 2012.

Evidence to recommendation. The panel agreed upon the proposed list and does not consider necessary any substantial modification.

External reviewers median score 9/10 ( $100 \%$ of scores $\geq 7$ ).

\section{RECOMMENDATION 5}

FMS should be diagnosed as a clinical construct without any confirmatory laboratory tests (level 5, grade D).

Repeated examinations after diagnosis should be avoided, unless guided by the onset of new symptoms or semeiotic findings (level 5, grade D).

Any further laboratory or radiographic analysis should depend on the individual patient's clinical evaluation, which may suggest some other medical condition (level 5, grade D).

Summary of guidelines. Both selected CPGs based exclusively the diagnosis on clinical manifestations of FMS. However, the diagnosis must be advanced by an expert clinician, who is confident in the differential diagnosis with other diseases (also through adequate laboratory or instrumental tests). These examinations should be repeated whenever there are changes in the clinical scenario that cannot be attributed to FMS.

Recommendation/supporting evidence. Canadian 2013, German 2012.

Evidence to recommendation. The panel agrees to identify in the rheumatologist the decision maker of the examinations to be performed in the context of the overall diagnostic process. Blood tests have a prominent role only for the differential diagnostics. If other diseases in association with FMS are suspected, the following tests should be considered: erythrocyte sedimentation rate (ESR), C-reactive protein (CRP), complete blood count, thyroid stimulating hormone (TSH), creatine phosphokinase (CPK), transaminases and $\gamma$-glutamyl transferase. Targeted tests can be required in case of additional signs or 
symptoms (30). In FMS there is a significant presence of small fiber neuropathy (A delta and C), but skin biopsy should be considered only in selected cases (33).

External reviewers median score $8 / 10$ ( $91 \%$ of scores $\geq 7$ ).

\section{RECOMMENDATION 6}

After FMS diagnosis, the patient should be informed about the recommended and non-recommended treatment measures (level 4, grade A).

Healthcare professionals should be educated about the pathogenesis, empathetic, open and honest, without negative attitudes and pursuing the realization of a shared decision-making process (level 3, grade D).

The patient should be informed that FMS is a disease characterized by alterations of the functional sphere and there is no clear evidence of organic damage. Healthcare professionals should confirm the legitimacy of the disorder, provide information on the long-term prognosis (i.e. quoad-vitam assessment) and clearly explain the symptoms to the patient (level 3 , grade D).

The patient's ability to alleviate these symptoms through coping activities and strategies should be emphasized, along with the fact that the outcome is often favorable, even if the symptoms may vary over time (level 3, grade B).

Patient associations may play an active role in promoting educational and support activities (e.g. self-help groups, information material) (level 5, grade D).

Summary of guidelines. All the selected guidelines agree on the opportunity to advise and adequately inform the patient about FMS nature and prognosis. This aspect is crucial in order to build and preserve the doctor-patient alliance and the subsequent therapeutic path.

Recommendation/supporting evidence. Emilia-Romagna 2017, Canadian 2013, German 2012.

Evidence to recommendation. Experts point out that patients should be properly trained on FMS. It is mandatory to explain how much FMS is characterized by nociplastic pain. According to the International Association for the Study of the Pain (IASPPAIN www.iasp-pain.org) definition, nociplastic pain derives from alteration of nociception, although there is no clear evidence of tissue damage causing the activation of peripheral nociceptors or evidence of disease or lesion of the somatosensory system that triggers pain.

The panel of experts underlines the importance of patient support and education activities, that, in patients with mild disease, make the therapeutic aims achievable for every patient.

External reviewers median score 9/10 ( $91 \%$ of scores $\geq 7$ ).

\section{RECOMMENDATION 7}

Physicians should choose the pharmacological strategy (including drugs' combination) according to symptoms and paying particular attention to drug interactions (level 5, grade D).

Pharmacological treatments should be started at low doses with a progressive and prudent increase in the dosage, in order to reduce the poor tolerance and the appearance of possible side effects (level 5 , grade D).

In more complex cases, poorly responsive to standard therapies, it is advisable to adopt a 'multi-modal' approach (physical activity with at least psychotherapeutic support), to be shared with the patient (level 1, grade A).

Summary of guidelines. Of the selected CPGs, only the Canadian ones introduce the treatment strategy theme, reporting it as a recommendation.

Recommendation/supporting evidence. Canadian 2013.

Evidence to recommendation. The panel of experts confirms the attitude proposed by the Canadian CPGs focusing on treatment combination, and in particular on the multi-modal approach. Furthermore, a cautious attitude on the dose of the drugs used and on the evaluation of possible interactions is advisable. 
External reviewers median score $8 / 10$ ( $75 \%$ of scores $\geq 7$ ).

\section{RECOMMENDATION 8}

According to WHO step-up analgesic scale, paracetamol is useful in some patients; clinicians should be aware of the dose necessary to the pharmacological effect and the dose causing a toxic effect (level 5, grade D).

A therapeutic attempt with weak opioids, especially tramadol (level 1 , grade A), should be reserved for patients with moderate to severe pain who do not respond to other treatment modalities (level 2 , grade D).

Clinicians should closely monitor pharmacological action, with particular reference to drug-dependent side effects or behavioral disorders (level 5, grade D).

Opioid use should be discouraged in the absence of symptoms improvement (level 5, Grade D).

Summary of guidelines. Two CPGs recommend the cautious use of analgesics, paying attention to the effective dose and side effects. The analgesic with the strongest evidence of effectiveness is tramadol.

Recommendation/supporting evidence. EULAR 2016, Canadian 2013.

Evidence to recommendation. The panel agrees that the use of analgesics is not sufficiently supported by the literature; even for new molecules, such as tapentadol, which seemed to show an acceptable safety profile (34), there is a lack of robust evidence about the efficacy in FMS (35). Therefore, also for their side effects, it is advisable to avoid using analgesics, especially if there is not any improvement.

External reviewers median score $8 / 10$ ( $92 \%$ of scores $\geq 7$ ).

\section{RECOMMENDATION 9}

Anticonvulsant drugs, in particular pregabalin, are useful for their pain modulating properties; treatment should start with the lowest possible dose, followed by a progressive increase in dosage, with attention to adverse events (level 1, grade A).
Summary of guidelines. The use of pregabalin is recommended by all the CPGs examined. The doses are not expressly indicated, although it is stressed that the dosage should be progressively increased in order to reach the maximum tolerated one. Recommendation/supporting evidence. EULAR 2016, Canadian 2013, German 2012. Evidence to recommendation. The panel of experts confirms the use of pregabalin in FMS treatment. Although it is not easy to generalize, the starting dose may be between 50 and $75 \mathrm{mg}$. The maximum dosage should be around $300 \mathrm{mg}$ per day, divided into two administrations: the evening dose may be higher in order to exploit the hypno-inductive action. Higher doses may increase the frequency of side effects without further benefits (36).

External reviewers median score $8 / 10$ ( $75 \%$ of scores $\geq 7$ ).

\section{RECOMMENDATION 10}

In FMS treatment, serotonin reuptake inhibitors (fluoxetine and paroxetine) and norepinephrine (duloxetine) or tricyclic antidepressant drugs (amitriptyline) (level 1, grade A) may be used.

Summary of guidelines. The CPGs agree in identifying only some tricyclic antidepressants and some selective inhibitors of serotonin and norepinephrine reuptake for the treatment of FMS.

Recommendation/supporting evidence. EULAR 2016, Canadian 2013, German 2012.

Evidence to recommendation. Although the use of these drugs is widely supported by the literature, the panel suggests that they should be used only under the close supervision of physicians experienced with these treatments. In particular, for the association between drugs and the management of side effects, it is recommended to collaborate with a psychiatrist.

External reviewers median score 9/10 ( $83 \%$ of scores $\geq 7$ ).

\section{RECOMMENDATION 11}

Treatment with cyclobenzaprine (level 1, grade A) and cannabinoids (level 3, grade 
C) may be considered in FMS patient, especially in the context of significant sleep disturbances.

Summary of guidelines. The EULAR guidelines identify cyclobenzaprine as an effective choice for sleep disorders. Canadian CPGs propose, albeit with weak evidence, the possible use of cannabinoids for sleep disorders in FMS patients.

Recommendation/supporting evidence. EULAR 2016, Canadian 2013.

Evidence to recommendation. The use of these drugs is poorly supported by the literature. The majority of studies concern nabilone, a pure synthetic cannabinoid, which did not show to be effective in treating FMS symptoms. Cannabis, on the other hand, is a very complex phytopharmaceutical compound, including other types of cannabinoids and other substances such as terpenes, and it showed to be effective in FMS chronic pain and sleep disturbances treatment in preliminary studies (37-39).

However, the overall path to cannabinoid treatment has not been yet clearly defined in Italy, as well as the dispensation issues, the preparation sites, and the follow-up of FMS patients.

External reviewers median score $7 / 10$ ( $92 \%$ of scores $\geq 7$ ).

\section{RECOMMENDATION 12}

Non-pharmacological strategies providing for the patients' active participation should be an integral part of FMS therapeutic approach (level 1, grade A).

Physicians should encourage patients to pursue a regular lifestyle, gradually increasing physical activity through stimuli and/or to maintain or improve function and promote self-management by multimodal therapy (level 4, grade D).

Physicians should inform patients of the negative impact that the psychological distress associated with FMS can generate (level 3, grade D) and therefore promote psychological assessment or counseling (level 5, grade C).

Summary of guidelines. All the guidelines, and in particular the Canadian CPGs, un- derlined the paramount role of associating a non-pharmacological strategy to the treatment of FMS patients.

Recommendation/supporting evidence. Canadian 2013.

Evidence to recommendation. The multimodal approach significantly increases the improvement related to FMS treatment. The panel agreed on highlighting its clinical relevance, as well as the importance of addressing the psycho-affective component. Therefore, a psychological evaluation is recommended, in agreement with patients' perspective.

External reviewers median score 8/10 ( $92 \%$ of scores $\geq 7$ ).

\section{RECOMMENDATION 13}

Physical therapies (adapted to patient individual performance level) should be considered in FMS, such as aerobic resistance training; strengthening exercise; water activity/water jogging; thermal therapy (bath in thermal springs) (level 1 , grade $\mathrm{A}$ ).

Summary of guidelines. All CPGs agree to recommend physical activity that is supported by moderate evidence, while the German authors strongly support thermal therapy, more than those who drafted the EULAR recommendations.

Recommendation/supporting evidence. Emilia-Romagna 2017, EULAR 2016, Canadian 2013, German 2012.

Evidence to recommendation. Patient tailored exercise is considered an essential part of FMS therapy. Therefore, the panel agreed to endorse this recommendation. Opinions about thermal therapy are discordant. As thermal therapy is acknowledged as part of the Italian Health System, and taking into account the ease of access to the thermal structures, this intervention can be considered as a therapeutic option (40, 41). Furthermore, the great variety of exercises and strength training effective in FMS plays a fundamental role in the treatment strategy (42).

External reviewers median score 9/10 ( $83 \%$ of scores $\geq 7$ ). 


\section{RECOMMENDATION 14}

Psychological therapies to be considered may include:

a) behavioral-cognitive therapy and occupational therapy, including patient education, even for a short period (level 1, grade A);

b) hypnosis, guided imagination or therapeutic writing (level 3, grade C).

Summary of guidelines. All CPGs agree to recommend psychological therapy with a high level and strength of recommendation.

Recommendation/supporting evidence. Emilia-Romagna 2017, EULAR 2016, Canadian 2013, German 2012.

Evidence to recommendation. The panel agreed in stressing the importance of psychological therapy in terms of cognitivebehavioral therapy. This type of treatment encompasses some techniques such as hypnosis or therapeutic writing, which showed a significant advantage in association with drug treatment.

External reviewers median score 9/10 ( $91 \%$ of scores $\geq 7$ ).

\section{RECOMMENDATION 15}

Non-conventional therapies to be considered may be:

- meditative movement therapies (qigong, yoga, tai chi), mindfulnessbased stress reduction program and relaxation training (combined with exercise) (level 1, grade A).

- acupuncture (level 1, grade A).

- hydrotherapy (level 1, grade C).

Summary of guidelines. Only the EULAR guidelines include alternative therapies in the treatment of FMS although they are not adequately supported by the literature.

Recommendation/supporting evidence. EULAR 2016.

Evidence to recommendation. The panel confirmed this approach in a multi-modal context, paying attention to the process of patient's awareness about the disease, also thanks to adequate diversified support as needed.

External reviewers median score $8 / 10$ (83\% of scores $\geq 7$ ).

\section{RECOMMENDATION 16}

Clinical follow-up should rely on the judgment of the rheumatologist, with more frequent visits during the initial phase or until the symptoms' stabilization (level 5, grade E).

The development of a new symptom may require a clinical evaluation in order to exclude another disease (level 5, grade $\mathrm{E})$.

atient objectives and achievement levels should be recorded as a useful strategy for outcome (level 5, grade E).

Physicians should take into consideration that factors such as passivity, lack of self-control and mood disturbance may negatively influence the outcomes (level 5 , grade E).

Tender points examination should not be an outcome measure (level 3, grade C).

Summary of guidelines. The selected guidelines do not agree in indicating which tools to use in FMS follow-up. Narrative symptoms reports, generic subjective changes' assessment, specific (i.e. Revised Fibromyalgia Impact Questionnaire - FIQ-R) or generic (i.e. European Quality of Life - EQ-5D) questionnaires can be useful tools. In particular the FIQ$\mathrm{R}$ (FMS) assesses FMS impact on health and quality of life, both at baseline and during follow-up.

Recommendation/supporting evidence. Emilia-Romagna 2017, Canadian 2013.

Evidence to recommendation. The panel does not support the use of the European Quality of Life (EQ-5D) because it is not validated in FMS. On the other hand, the Fibromyalgia Assessment Status (FAS) is more diffused in clinical practice. FAS is validated in FMS, showing high sensitivity and diagnostic specificity, excellent clinimetric properties as it explores the domains relevant for physicians and patients (30).

The rheumatologist role in monitoring complex cases with comorbidities or assessing failure to the first line of treatment is pivotal.

External reviewers median score: 8/10 $(60 \%$ of scores $\geq 7)$. 


\section{RECOMMENDATION 17}

Treatment benefits should be regularly evaluated by patients and physicians as therapy should be continued only if positive effects are experienced. In the case of response to drug therapy, after an appropriate duration of treatment, a gradual withdrawal (level 2, grade $\mathrm{A}$ ) should be considered.

Patients who experience improvement with aerobic endurance training should constantly implement it (level 1, grade A). Physicians should encourage patients to work providing, if necessary, recommendations about maintaining the optimal productivity as the outcome is generally more favorable for those who are employed (level 3, grade D).

FMS patients on long-term sick leave should join appropriate rehabilitation program aimed at improving function, including returning to work if possible (level 5, grade D).

For long-term therapy, procedures that can be carried out independently (e.g., individual adaptation to resistance and/or strength training, stretching or heat therapy) should be self-managed by patients (level 2 grade A).

Summary of guidelines. The CPGs on which the recommendation is based agree in stressing the importance of verifying the benefits following the adoption of adequate physical therapy, including the self-managed one. The aim is to avoid leaving job and to stop drug therapy. The time interval between these assessments and the most appropriate professional figure who should carry them out is still not clearly identified. Recommendation/supporting evidence. Emilia-Romagna 2017, Canadian 2013, German 2012.

Evidence to recommendation. The positive effect of therapy must be intended as a significant improvement in the clinimetric scores. In particular, according to the Fibromyalgia Working Group of the Italian Ministry of Health, the positive effect is defined as:

a) a $30 \%$ FIQ-R score improvement (compared to baseline);

b) the achievement of remission (FIQ score $<30$ ); c) mild disease activity (FIQ score <45) (42). The appropriateness of the duration of drug treatment is established by the physician in consultation with the patient.

External reviewers median score: 9/10 ( $100 \%$ of scores $\geq 7$ ).

\section{DISCUSSION}

The support of SIR aiming at the development of CPGs on FMS management was mainly due to the absence of homogeneous recommendations adapted to the national scenario. Although FMS is a disease characterized by a high prevalence in the general population and with a significant socioeconomic burden for the National Health System and for individual patients, there have not been CPGs covering the protean aspects of this disease so far. The need to draw up shared guidelines faces various difficulties. One of the most important is the identification of the experts of the disease (who belong to extremely heterogeneous disciplines such as Rheumatology, Physiatry, Psychology, Psychiatry, Neurology, Algology, etc.). Moreover, diagnostic and therapeutic aspects are extremely variable as they are affected by the socio-economic contexts.

The effort to make FMS management homogeneous and evidence-based is as crucial as the need to provide patients suffering from severe and disabling disease with the possibility of a recognized support (30). For this reason, the SIR has established, on behalf of the Ministry of Health, the first national register on FMS (30) and it is currently engaged in identifying the levels of disease severity and evaluating the response to treatment (43).

These CPGs are meant to be a useful tool for the management of patients with FMS. A countrywide homogeneous FMS management will improve both daily practice and clinical/epidemiological researches.

A number of limitation may be found in these adapted CPG. First, the most recent clinical studies which may have an impact on clinical practice were not systematically considered in the process of adaptation. For example, there is growing evidence 
Table IV - Summary of FMS pharmacological treatment option management with respective level of evidence (the green colored squares correspond to Level 1 Grade A; the yellow colored squares correspond to Level 3 Grade C; the orange colored squares correspond to Level 5 Grade D).

\begin{tabular}{|c|c|c|c|c|}
\hline \multicolumn{5}{|c|}{ PHARMACOLOGIC TREATMENT } \\
\hline Analgesic & Anticonvulsant & Antidepressants & Muscle relaxants & Others \\
\hline Opioids & \multirow{3}{*}{ Pregabalin } & Fluoxetine and paroxetine & \multirow{2}{*}{ Cyclobenzaprine } & \multirow{2}{*}{ Cannabinoids } \\
Paracetamol & & Duloxetine & \\
\cline { 3 - 3 } & & Amitriptyline & & \\
\hline
\end{tabular}

Table V - Summary of FMS non pharmacological treatment option management with respective level of evidence (the green colored squares correspond to Level 1 Grade A (*hydrotherapy level 1 grade C); the yellow colored squares correspond to Level 3 Grade C; the orange colored squares correspond to Level 5 Grade D).

\begin{tabular}{|c|c|c|}
\hline \multicolumn{2}{|c|}{ NON PHARMACOLOGIC TREATMENT } \\
\hline Physical therapies & Psychological therapies & Non-conventional therapies \\
\hline Aerobic resistance training & $\begin{array}{c}\text { Behavioral-cognitive therapy } \\
\text { and occupational therapy }\end{array}$ & $\begin{array}{c}\text { Meditative movement therapies } \\
\text { (qigong, yoga, tai chi) }\end{array}$ \\
\hline Strengthening exercise & Hypnosis & $\begin{array}{c}\text { Mindfulness-based stress reduction } \\
\text { program and relaxation training }\end{array}$ \\
\hline Water activity/water jogging & Guided imagination & Acupuncture \\
\hline Thermal therapy & Therapeutic writing & Hydrotherapy \\
\hline
\end{tabular}

about the use of cannabinoids in FMS with relevant sleep disturbances (44).

Secondly, the update of the source guidelines is limited and these adapted recommendations were based on original CPGs which were published in a 10-year time frame. Therefore, they consider established management and treatment strategies. On the other hand, they may appear more elusive on some issues (e.g. use of cannabinoids) (Tables IV and V).

To improve and standardize the management of FMS in Italy, the SIR plans to disseminate these recommendations through national conferences and educational courses for health professionals. Application and dissemination of these CPGs is expected to improve the treatment of FMS patients.

\section{Plans of update}

These recommendations were endorsed by SIR as guidelines only and they do not substitute the individual clinicians' judgment since they may not apply to all patients and all clinical situations. SIR plans to review and update these recommendations in the future to determine if they remain current and to capture future treatments or advances in FMS management.

\section{Conflict of interest}

The Authors do not disclose any conflict of interest.

\section{Acknowledgements}

The Authors acknowledge all the external reviewers (in alphabetical order those who gave consent for publication): Atzeni F, Batticciotto A, Di Franco M, El Aoufy K, Fabio G, Fusaro E, Gerardi MC, Leccabue M, Maddali Bongi S, Meani L, Stisi S, V De Giorgis. We are grateful to Solazzo T. as part of SIR secretariat for administrative support.

\section{Authors' Contributions}

All authors were involved in drafting the article or revising it critically for important intellectual content, and all authors approved the final version to be published. Conception and design: C.A.S., A.A., S.P., N.U., I.P., A.B., M.M., G.C., Systematic review search and $\mathrm{CPG}$ rating: A.A., S.P.. Analysis and discussion of the recommendations: A.A., S.P., P.SP., L.B., F.S., N.U., I.P., A.B., M.M., C.A.S. 


\section{REFERENCES}

1. Siracusa R, Paola RD, Cuzzocrea S, Impellizzeri D. Fibromyalgia: pathogenesis, mechanisms, diagnosis and treatment options update. Int J Mol Sci. 2021; 22: 3891.

2. Salaffi F, De Angelis R, Grassi W; Marche Pain Prevalence; INvestigation Group (MAPPING) study. Prevalence of musculoskeletal conditions in an Italian population sample: results of a regional community-based study. I. The MAPPING study. Clin Exp Rheumatol. 2005; 23: 819-828.

3. Clauw DJ. Fibromyalgia. A clinical review. JAMA. 2014; 311: 1547-55.

4. Jones GT, Atzeni F, Beasley M, et al. The prevalence of fibromyalgia in the general population. A comparison of the American College of Rheumatology 1990, 2010, and Modified 2010 Classification Criteria. Arthrit Rheumatol. 2015; 67: 568-575.

5. Harris RE, Clauw DJ, Scott DJ, et al. Decreased central mu-opioid receptor availability in fibromyalgia. J Neurosci. 2007; 27: 1000010006.

6. Kadetoff D, Lampa J, Westman M, et al. Evidence of central inflammation in fibromyalgia-increased cerebrospinal fluid interleukin-8 levels. J Neuroimmunol. 2012; 242: 33-38.

7. Watkins LR, Hutchinson MR, Rice KC, Maier SF. The 'toll' of opioid-induced glial activation: improving the clinical efficacy of opioids by targeting glia. Trends Pharmacol Sci. 2009; 30: 581-591.

8. Harris RE, Sundgren PC, Craig AD, et al. Elevated insular glutamate in fibromyalgia is associated with experimental pain. Arthritis Rheum. 2009; 60: 3146-3152.

9. Foerster BR, Petrou M, Edden RA, et al. Reduced insular gamma- aminobutyric acid in fibromyalgia. Arthritis Rheum. 2012; 64: 579-583.

10. Petrovic P, Kalso E, Petersson KM, Ingvar M. Placebo and opioid analgesia-- imaging a shared neuronal network. Science. 2002; 295 : 1737-1740.

11. Guedj E, Taieb D, Cammilleri S, et al. 99mTcECD brain perfusion SPECT in hyperalgesic fibromyalgia. Eur J Nucl Med Mol Imaging. 2007; 34: 130-134.

12. Gracely RH, Petzke F, Wolf JM, Clauw DJ. Functional magnetic resonance imaging evidence of augmented pain processing in fibromyalgia. Arthritis Rheum. 2002; 46: 1333-1343.

13. Feraco P, Bacci A, Pedrabissi F, et al. Metabolic abnormalities in pain-processing regions of patients with fibromyalgia: a $3 \mathrm{~T}$ MR spectroscopy study. AJNR Am J Neuroradiol. 2011; 32: 1585-1590.

14. Hauser W, Ablin J, Fitzcharles MA, et al. Fibromyalgia. Nat Rev Dis Primers. 2015: 15022.
15. Littlejohn G. Neurogenic neuroinflammation in fibromyalgia and complex regional pain syndrome. Nat Rev Rheumatol. 2015; 11: 639-648.

16. Uceyler N, Zeller D, Kahn AK, et al. Small fibre pathology in patients with fibromyalgia syndrome. Brain. 2013; 136: 1857-1867.

17. Goldenberg DL, Schur PH, Romain PL. Differential diagnosis of fibromyalgia. Literature review. UpToDate; 12 sept 2016.

18. Arnold LM, Clauw DJ, McCarberg BH. Improving the recognition and diagnosis of fibromyalgia. Concise review for clinicians. Mayo Clin Proc. 2011; 86: 457-464.

19. Fervers B, Burgers JS, Haugh MC, et al. Adaptation of clinical guidelines: literature review and proposition for a framework and procedure. Int J Qual Health Care. 2006; 18: 167-176.

20. Fervers B, Burgers JS, Voellinger R, et al. ADAPTE Collaboration. Guideline adaptation: an approach to enhance efficiency in guideline development and improve utilisation. BMJ Qual Saf. 2011; 20: 228-236.

21. AGREE II Users' Manual; 2013. Available from: https://www.agreetrust.org/wpcontent/uploads/2013/10/AGREE-II-UsersManual-and-23-item-Instrument_2009_UPDATE_2013.pdf

22. Oxford Centre for Evidence-based Medicine. Levels of Evidence; March 2009. Available from: http://www.cebm.net/oxford-centreevidence-based-medicine-levels-evidencemarch-2009/

23. Manara M, Ughi N, Ariani A, et al. Providing updated guidelines for the management of rheumatic diseases in Italy with the ADAPTE methodology: a project by the Italian Society for Rheumatology. Reumatismo. 2019; 71: 1-4.

24. Macfarlane GJ, Kronisch C, Dean LE, et al. EULAR revised recommendations for the management of fibromyalgia. Ann Rheum Dis. 2016; 76: 318-328.

25. Fitzcharles M-A, Ste-Marie PA, Goldenberg DL, et al. 2012 Canadian Guidelines for the diagnosis and management of fibromyalgia syndrome: executive summary. Vol. 18, Pain Research \& Management; 2013. pp. 119-126. doi: 10.1155/2013/918216

26. Eich W, Häuser W, Arnold B, et al. Arbeitsgemeinschaft der Wissenschaftlichen Medizinischen Fachgesellschaften. Das Fibromyalgiesyndrom. Allgemeine Behandlungsgrundsätze, Versorgungskoordination und Patientenschulung (Fibromyalgia syndrome. General principles and coordination of clinical care and patient education). Schmerz. 2012; 26: 268-75. [In German].

27. Eich W, Häuser W, Arnold B, et al; Arbeitsgemeinschaft der Wissenschaftlichen Med- 
izinischen Fachgesellschaften. Das Fibromyalgiesyndrom. Definition, Klassifikation, klinische Diagnose und Prognose (Fibromyalgia syndrome. Definition, classification, clinical diagnosis and prognosis). Schmerz. 2012; 26: 247-258. [In German].

28. Häuser W, Eich W, Herrmann M, et al. Fibromyalgia syndrome: classification, diagnosis, and treatment. Dtsch Arztebl Int 2009; 106: 383-391.

29. Direzione Generale Cura della Persona, Salute e Welfare regionale, dell'Agenzia Sanitaria e Sociale regionale, delle Aziende Sanitarie della Emilia-Romagna. Linee di Indirizzo per la diagnosi e il trattamento della Fibromialgia; 2018. Available from: https://salute.regione.emilia-romagna.it/ normativa-e-documentazione/linee-di-indirizzo/fibromialgia

30. Salaffi F, Farah S, Di Carlo M, et al. Italian Society of Rheumatology (SIR) and Ministero della Salute - Direzione Generale della Programmazione Sanitaria, Roma, Italy. The Italian Fibromyalgia Registry: a new way of using routine real-world data concerning patientreported disease status in healthcare research and clinical practice. Clin Exp Rheumatol. 2020; 38 (Suppl.) 123: 65-71.

31. Wolfe F, Clauw DJ, Fitzcharles MA, et al. 2016 Revisions to the 2010/2011 fibromyalgia diagnostic criteria. Semin Arthritis Rheum. 2016; 46: 319-329.

32. Salaffi F, Di Carlo M, Farah S, et al. Diagnosis of fibromyalgia: comparison of the 2011/2016 ACR and AAPT criteria and validation of the modified Fibromyalgia Assessment Status. Rheumatology (Oxford) 2020 Mar 24.

33. Grayston R, Czanner G, Elhadd K, et al. A systematic review and meta-analysis of the prevalence of small fiber pathology in fibromyalgia: Implications for a new paradigm in fibromyalgia etiopathogenesis. Semin Arthritis Rheum. 2019; 48: 933-940.

34. Santos J, Alarcão J, Fareleira F, et al. Tapentadol for chronic musculoskeletal pain in adults. Cochrane Database Syst Rev. 2015; (5): CD009923.

35. Littlejohn GO, Guymer EK, Ngian GS. Is there a role for opioids in the treatment of fibromyalgia? Pain Manag. 2016; 6: 347-355.

36. Derry S, Cording M, Wiffen PJ, et al. Pregabalin for pain in fibromyalgia in adults. Cochrane Database Syst Rev. 2016; 9: CD011790.

37. Sarzi-Puttini P, Ablin J, Trabelsi A, et al. Cannabinoids in the treatment of rheumatic diseases: Pros and cons. Autoimmun Rev. 2019; 18: 102409.

38. Sarzi-Puttini P, Batticciotto A, Atzeni F, et al. Medical cannabis and cannabinoids in rheumatology: where are we now? Expert Rev Clin Immunol. 2019; 15: 1019-1032.

39. Giorgi V, Bongiovanni S, Atzeni F, et al. Adding medical cannabis to standard analgesic treatment for fibromyalgia: a prospective observational study. Clin Exp Rheumatol. 2020; 38 Suppl 123: 53-59.

40. Pérez-Fernández MR, Calvo-Ayuso N, Martínez-Reglero C, et al. Efficacy of baths with mineral-medicinal water in patients with fibromyalgia: a randomized clinical trial. Int J Biometeorol. 2019; 63: 1161-1170.

41. Bazzichi L, Da Valle Y, Rossi A, et al. A multidisciplinary approach to study the effects of balneotherapy and mud-bath therapy treatments on fibromyalgia. Clin Exp Rheumatol. 2013; 31: S111-120.

42. Andrade A, Hech Dominski F, Mendes Sieczkowska $S$. What we already know about the effects of exercise in patients with fibromyalgia: An umbrella review. Semin Arthrit Rheum. 2020; 50: 1465-1480.

43. Salaffi F, Bazzichi L, Atzeni F, et al. Characterization and definition of disease severity status in fibromyalgia: results from a cross-sectional survey of 2,339 Italian people. Rheumatology (Oxford) 2021; 60: 728-736.

44. Sarzi-Puttini P, Batticciotto A, Atzeni F, et al. Medical cannabis and cannabinoids in rheumatology: where are we now? Expert Rev Clin Immunol. 2019; 15: 1019-1032. 